\title{
Eigenvalue estimates for the Aharonov-Bohm operator in a domain
}

\author{
Rupert L. Frank and Anders M. Hansson
}

\begin{abstract}
We prove semi-classical estimates on moments of eigenvalues of the Aharonov-Bohm operator in bounded two-dimensional domains. Moreover, we present a counterexample to the generalized diamagnetic inequality which was proposed by Erdős, Loss and Vougalter. Numerical studies complement these results.
\end{abstract}

\section{Introduction}

We shall study inequalities for the eigenvalues of the Aharonov-Bohm operator

$$
H_{\alpha}^{\Omega}:=\left(\mathbf{D}-\alpha \mathbf{A}_{0}\right)^{2} \quad \text { in } L_{2}(\Omega) .
$$

Here $\Omega \subset \mathbb{R}^{2}$ is a bounded domain, $\mathbf{D}:=-i \nabla$ and $\alpha \mathbf{A}_{0}(x):=\alpha|x|^{-2}\left(-x_{2}, x_{1}\right)^{T}$ is a vector potential generating an Aharonov-Bohm magnetic field with flux $\alpha$ through the origin. We shall assume that this point belongs to the interior of the simply-connected hull of $\Omega$ and that $\alpha \notin \mathbb{Z}$, for otherwise $\alpha \mathbf{A}_{0}$ can be gauged away. On the boundary of $\Omega$ we impose Dirichlet boundary conditions. More precisely, the operator (1.1) is defined through the closure of the quadratic form $\left\|\left(\mathbf{D}-\alpha \mathbf{A}_{0}\right) u\right\|^{2}$ on $C_{0}^{\infty}(\Omega \backslash\{0\})$.

Before stating our main results we would like to recall some well-known semiclassical spectral asymptotics and estimates for the Dirichlet Laplacian $-\Delta^{\Omega}$ and its magnetic version $(\mathbf{D}-\mathbf{A})_{\Omega}^{2}$, $\mathbf{A}$ being an arbitrary vector potential. If $\Omega$ is bounded then the spectrum of $-\Delta^{\Omega}$ is discrete, and by a classical result due to Weyl (see, e.g., [ReS]) one has, as $\Lambda \rightarrow \infty$,

$$
\operatorname{tr}\left(-\Delta^{\Omega}-\Lambda\right)_{-}^{\gamma} \sim \frac{1}{(2 \pi)^{2}} \iint_{\Omega \times \mathbb{R}^{2}}\left(|\xi|^{2}-\Lambda\right)_{-}^{\gamma} d x d \xi=\frac{1}{4 \pi(\gamma+1)}|\Omega| \Lambda^{\gamma+1}
$$

for all $\gamma \geq 0$. Note that the right-hand side involves the symbol $|\xi|^{2}$ on the phase space $\Omega \times \mathbb{R}^{2}$. The asymptotics (1.2) are accompanied by the estimate

$$
\operatorname{tr}\left(-\Delta^{\Omega}-\Lambda\right)_{-}^{\gamma} \leq R_{\gamma} \frac{1}{(2 \pi)^{2}} \iint_{\Omega \times \mathbb{R}^{2}}\left(|\xi|^{2}-\Lambda\right)_{-}^{\gamma} d x d \xi, \quad \gamma \geq 0
$$

Received by the editors February 5, 2007. 
with a universal constant $R_{\gamma}$ independent of $\Omega$ and $\Lambda$, and one is interested in the sharp value of this constant $R_{\gamma}$. In view of (1.2) the sharp constant obviously cannot be smaller than 1, and by an argument of Aizenman and Lieb [AL, it is a non-increasing function of $\gamma$. Pólya $[\mathrm{P}]$ proved the estimate (1.3) for $\gamma=0$ with constant 1 under the additional assumption that $\Omega$ is a tiling domain. His famous conjecture that this is true for arbitrary domains is still unproved. Berezin B1] and independently Li and Yau [LiY] (see also [La]) proved (1.3) for $\gamma \geq 1$ with the sharp constant $R_{\gamma}=1$. This also yields the so far best known bound on the sharp constant for $\gamma=0$, namely $R_{0} \leq 2$. Indeed,

$$
\operatorname{tr}\left(-\Delta^{\Omega}-\Lambda\right)_{-}^{0} \leq(\mu-\Lambda)^{-1} \operatorname{tr}\left(-\Delta^{\Omega}-\mu\right)_{-} \leq(\mu-\Lambda)^{-1} \frac{1}{8 \pi}|\Omega| \mu^{2}, \quad \mu>\Lambda,
$$

and the claim follows by optimization with respect to $\mu$. We note that the estimate (1.3) for $\gamma=0$ and $\gamma=1$ is closely related to the estimates

$$
\lambda_{N}^{\Omega} \geq \rho_{0} 4 \pi|\Omega|^{-1} N \quad \text { and } \quad \sum_{j=1}^{N} \lambda_{j}^{\Omega} \geq \rho_{1} 2 \pi|\Omega|^{-1} N^{2}
$$

for the eigenvalues $\lambda_{j}^{\Omega}$ of the operator $-\Delta^{\Omega}$. The form (1.3), however, shows the close connection with the Lieb-Thirring inequality, see [LT] and also the review article LaW2.

We now turn to the 'magnetic' analog of (1.3), i.e., where $-\Delta^{\Omega}$ is replaced by the Dirichlet realization of the operator $(\mathbf{D}-\mathbf{A})_{\Omega}^{2}$ in $L_{2}(\Omega)$ and $\mathbf{A}$ is a (sufficiently regular) magnetic vector potential. Note that the value of the right-hand side in (1.3) does not change if $\xi$ is replaced by $\xi-\mathbf{A}(x)$. Hence one is interested in the estimate

$$
\operatorname{tr}\left((\mathbf{D}-\mathbf{A})_{\Omega}^{2}-\Lambda\right)_{-}^{\gamma} \leq R_{\gamma}^{\operatorname{mag}} \frac{1}{(2 \pi)^{2}} \iint_{\Omega \times \mathbb{R}^{2}}\left(|\xi|^{2}-\Lambda\right)_{-}^{\gamma} d x d \xi, \quad \gamma \geq 0
$$

with a universal constant $R_{\gamma}^{\text {mag }}$ independent of $\Omega, \Lambda$ and $\mathbf{A}$. It is a consequence of the sharp Lieb-Thirring inequality by Laptev and Weidl [LaW1] that $R_{\gamma}^{\text {mag }}=1$ for $\gamma \geq \frac{3}{2}$. Not much is known about (1.5) in the case $\gamma<\frac{3}{2}$. The Laptev-Weidl result and an argument similar to (1.4) yield the (probably non-sharp) estimate $R_{\gamma}^{\mathrm{mag}} \leq\left(\frac{5}{3}\right)^{3 / 2}(\gamma /(\gamma+1))^{\gamma}$ for $\gamma<\frac{3}{2}$. For $\gamma=0$ and $\gamma=1$ in particular one finds the values 2.1517 and 1.0758, respectively. In [ELoV] the estimate (1.5) is shown to hold for $\gamma \geq 1$ with constant 1 in the special case of a homogeneous magnetic field

$$
\mathbf{A}(x)=\frac{B}{2}\left(-x_{2}, x_{1}\right)^{T}
$$

It was recently shown in [FLoW that (1.5) does not hold with constant 1 if $0 \leq \gamma<1$, not even when $\Omega$ is tiling. Moreover, the authors determined the optimal constant such that (1.5) holds for all $\Lambda$ under the constraint that $\mathbf{A}$ is given by (1.6) and $\Omega$ is tiling. 
In this paper we shall consider A corresponding to an Aharonov-Bohm magnetic field and we shall prove the estimate

$$
\operatorname{tr}\left(H_{\alpha}^{\Omega}-\Lambda\right)_{-}^{\gamma} \leq C_{\gamma}(\alpha) \frac{1}{(2 \pi)^{2}} \iint_{\Omega \times \mathbb{R}^{2}}\left(|\xi|^{2}-\Lambda\right)_{-}^{\gamma} d x d \xi, \quad \gamma \geq 1
$$

with a constant $C_{\gamma}(\alpha)$ given explicitly in terms of Bessel functions. Even though our bound is probably not sharp, it improves upon the previously known estimates. Indeed, numerical evaluation of our constant shows that (1.7) holds for all $\alpha$ with constants $C_{0}(\alpha)=1.0540$ and $C_{1}(\alpha)=1.0224$ if $\gamma=0$ and 1 , respectively, see Section 4. We complement our analytical results with a numerical study of the eigenvalue of the operator (1.1) for five domains: a disc, a square and three different annuli. In all cases the estimate (1.7) seems to be valid with constant 1 . We refer to Section [5] and Figures 1010 for a detailed account of the outcome of our experiments.

For the proof of our eigenvalue estimate we proceed similarly as in ELoV]. Indeed, by the Berezin-Lieb inequality, (1.7) is an immediate consequence of the generalized diamagnetic inequality

$$
\operatorname{tr} \chi_{\Omega}\left(H_{\alpha}-\Lambda\right)_{-}^{\gamma} \chi_{\Omega} \leq R_{\gamma}(\alpha) \operatorname{tr} \chi_{\Omega}(-\Delta-\Lambda)_{-}^{\gamma} \chi_{\Omega} \quad \text { for all bounded } \Omega \subset \mathbb{R}^{2} \text {. }
$$

Here $H_{\alpha}:=H_{\alpha}^{\mathbb{R}^{2}}$ denotes the Aharonov-Bohm operator in the whole space. In ELoV an analogous estimate was proved in the case (1.6) with constant 1 when $\gamma \geq 1$. The authors conjectured that such an inequality is not true for an arbitrary magnetic field, but their counterexample contains a gap; the condition 2) on p. 905 can not be satisfied by a non-trivial radial vector field, as it was first pointed out by M. Solomyak. This gap can be removed by a minor change in the argument, since the assumption of radial symmetry is not essential in the proof [ELo. Nevertheless, we feel that our example is of independent interest and sheds some light on the particularities of the Aharonov-Bohm operator. What we prove is that the sharp constant in (1.8) is strictly greater than unity, see Theorem 3.1. We establish this by a thorough study of the local spectral density of the operator $H_{\alpha}$, see Section 2 ,

We mention in closing the papers BaEvLe, EkF], [H], [MOR, where LiebThirring estimates for the Schrödinger operator $H_{\alpha}+V$ were obtained. Our estimates can be seen as a refinement of these estimates in the special case where the potential $V$ is equal to a negative constant $-\Lambda$ inside and equal to infinity outside a bounded domain $\Omega \subset \mathbb{R}^{2}$.

Acknowledgements. The authors would like to thank A. Laptev for the setting of the problem and helpful remarks. The first author is grateful to E. H. Lieb and R. Seiringer for their hospitality at Princeton University and thanks them, H. Kalf and M. Loss for fruitful discussions. 


\section{The Aharonov-Bohm operator in the whole space}

\subsection{Diagonalization}

In this section we recall some well-known facts about the Aharonov-Bohm operator in the whole space, see, e.g., $\mathrm{AhBo}, \mathrm{Ru}$. We denote by $H_{\alpha}$ the self-adjoint operator in $L_{2}\left(\mathbb{R}^{2}\right)$ associated with the closure of the quadratic form

$$
\int_{\mathbb{R}^{2}}\left|\left(\mathbf{D}-\alpha \mathbf{A}_{0}\right) u\right|^{2} d x, \quad u \in C_{0}^{\infty}\left(\mathbb{R}^{2} \backslash\{0\}\right) .
$$

Here $\mathbf{D}:=-i \nabla, \mathbf{A}_{0}(x):=|x|^{-2}\left(-x_{2}, x_{1}\right)^{T}$ and $\alpha \in \mathbb{R}$. Moreover, $J_{\nu}$ denotes, as usual, the Bessel function of the first kind of order $\nu$, see AbSt. With polar coordinates $x=|x|\left(\cos \theta_{x}, \sin \theta_{x}\right)$ and similarly for $\xi$, we define

$$
\mathcal{F}_{\alpha}(\xi, x):=\frac{1}{2 \pi} \sum_{n \in \mathbb{Z}} J_{|n-\alpha|}(|\xi||x|) e^{i n\left(\theta_{\xi}-\theta_{x}\right)}, \quad \xi, x \in \mathbb{R}^{2},
$$

and put

$$
\left(\mathcal{F}_{\alpha} u\right)(\xi):=\int_{\mathbb{R}^{d}} \mathcal{F}_{\alpha}(\xi, x) u(x) d x, \quad \xi \in \mathbb{R}^{2},
$$

for $u \in C_{0}^{\infty}\left(\mathbb{R}^{2}\right)$. Note that $\mathcal{F}_{0}(\xi, x)=(2 \pi)^{-1} e^{-i \xi \cdot x}$ [AbSt, 9.1.41], so $\mathcal{F}_{0}$ is the ordinary Fourier transform, which diagonalizes $H_{0}=-\Delta$. Similarly, one has

Lemma 2.1. For any $\alpha \in \mathbb{R}, \mathcal{F}_{\alpha}$ extends to a unitary operator in $L_{2}\left(\mathbb{R}^{2}\right)$ and diagonalizes $H_{\alpha}$, i.e.,

$$
\left(\mathcal{F}_{\alpha} f\left(H_{\alpha}\right) u\right)(\xi)=f\left(|\xi|^{2}\right)\left(\mathcal{F}_{\alpha} u\right)(\xi), \quad \xi \in \mathbb{R}^{2},
$$

for any $u \in L_{2}\left(\mathbb{R}^{2}\right)$ and $f \in L_{\infty}(\mathbb{R})$.

We sketch a proof of this assertion for the sake of completeness.

Proof. The orthogonal decomposition

$$
L_{2}\left(\mathbb{R}^{2}\right)=\bigoplus_{n \in \mathbb{Z}} \mathfrak{H}_{n}, \quad \mathfrak{H}_{n}:=\left\{|x|^{-1 / 2} g(|x|) e^{i n \theta_{x}}: g \in L_{2}\left(\mathbb{R}_{+}\right)\right\},
$$

reduces $H_{\alpha}$. The part of $H_{\alpha}$ in $\mathfrak{H}_{n}$ is unitarily equivalent to the operator

$$
h_{|n-\alpha|}:=-\frac{d^{2}}{d r^{2}}+\frac{(n-\alpha)^{2}-1 / 4}{r^{2}} \quad \text { in } L_{2}\left(\mathbb{R}_{+}\right),
$$

which is defined as the Friedrichs extension of the corresponding differential expression on $C_{0}^{\infty}\left(\mathbb{R}_{+}\right)$. (We emphasize that in our notation $\mathbb{R}_{+}$means the open interval $(0, \infty)$.) The operator

$$
\left(\Phi_{\nu} g\right)(k):=\int_{\mathbb{R}_{+}} \sqrt{r k} J_{\nu}(r k) g(r) d r, \quad k \in \mathbb{R}_{+},
$$

initially defined for $g \in C_{0}^{\infty}\left(\mathbb{R}_{+}\right)$, extends to a unitary operator in $L_{2}\left(\mathbb{R}_{+}\right)$and diagonalizes $h_{\nu}$, i.e.,

$$
\left(\Phi_{\nu} f\left(h_{\nu}\right) g\right)(k)=f\left(k^{2}\right)\left(\Phi_{\nu} g\right)(k), \quad k \in \mathbb{R}_{+},
$$


for any $g \in L_{2}\left(\mathbb{R}_{+}\right)$and $f \in L_{\infty}(\mathbb{R})$ Ti, Ch. VIII]. The assertion of the lemma is a simple consequence of these facts.

The proof of the preceding lemma shows in particular that the operators $H_{\alpha}$ and $H_{\alpha+m}$ with $m \in \mathbb{Z}$ are unitarily equivalent via multiplication by $e^{i m \theta_{x}}$ (a gauge transformation). Hence, without loss of generality we shall assume that $0 \leq \alpha<1$.

Lemma 2.1 implies that $f\left(H_{\alpha}\right)$, at least formally, is an integral operator with integral kernel

$$
\begin{aligned}
f\left(H_{\alpha}\right)(x, y) & =\int_{\mathbb{R}^{2}} \overline{\mathcal{F}_{\alpha}(\xi, x)} f\left(|\xi|^{2}\right) \mathcal{F}_{\alpha}(\xi, y) d \xi \\
& =\frac{1}{2 \pi} \sum_{n \in \mathbb{Z}} \int_{0}^{\infty} f\left(k^{2}\right) J_{|n-\alpha|}(k|x|) J_{|n-\alpha|}(k|y|) e^{i n\left(\theta_{x}-\theta_{y}\right)} k d k \\
& =\frac{1}{4 \pi} \sum_{n \in \mathbb{Z}} \int_{0}^{\infty} f(\lambda) J_{|n-\alpha|}(\sqrt{\lambda}|x|) J_{|n-\alpha|}(\sqrt{\lambda}|y|) e^{i n\left(\theta_{x}-\theta_{y}\right)} d \lambda .
\end{aligned}
$$

On the diagonal this is

$$
f\left(H_{\alpha}\right)(x, x)=\frac{1}{4 \pi} \int_{0}^{\infty} f(\lambda) \rho_{\alpha}(\sqrt{\lambda}|x|) d \lambda
$$

where

$$
\rho_{\alpha}(t):=\sum_{n \in \mathbb{Z}} J_{|n-\alpha|}^{2}(t), \quad t \geq 0 .
$$

In particular, $\frac{1}{4 \pi} \rho_{\alpha}(\sqrt{\lambda}|x|)$ is the local spectral density at energy $\lambda$. In the following subsection we collect some basic information about this function, and in Subsections 2.3 and 2.5 we prove some results about the precise asymptotic behavior of (2.1) as $|x| \rightarrow \infty$ for special choices of functions $f$. This will allow us to prove that the generalized diamagnetic inequality is violated.

\subsection{The spectral density}

Our results are based on a detailed study of the function $\rho_{\alpha}$ from (2.2). We note that if $\alpha=0$ then $\rho_{0} \equiv 1$ by [AbSt, 9.1.76]. An expression in terms of elementary functions is also available AbSt, 5.2.15] for $\alpha=1 / 2$,

$$
\rho_{1 / 2}(t)=\frac{2}{\pi} \int_{0}^{2 t} \frac{\sin s}{s} d s
$$

As $t \rightarrow \infty, \rho_{1 / 2}(t)$ tends to 1 in an oscillating manner. As we shall see, this behavior appears for all $0<\alpha<1$. The starting point of our study of $\rho_{\alpha}$ with non-trivial flux $\alpha$ is the following

Lemma 2.2. For any $0<\alpha<1, \rho_{\alpha}$ is a smooth function on $\mathbb{R}_{+}$with $\rho_{\alpha}(0)=0$, $\rho_{\alpha}(t) \rightarrow 1$ as $t \rightarrow \infty$ and

$$
\rho_{\alpha}^{\prime}(t)=J_{\alpha}(t) J_{\alpha-1}(t)+J_{1-\alpha}(t) J_{-\alpha}(t), \quad t \geq 0 .
$$


Proof. By [Lu, 11.2(10)] and [AbSt, 11.4.42] one has, for all $t \geq 0$,

$$
\begin{aligned}
\rho_{\alpha}(t) & =\int_{0}^{t}\left(J_{\alpha}(s) J_{\alpha-1}(s)+J_{1-\alpha}(s) J_{-\alpha}(s)\right) d s \\
& =1-\int_{t}^{\infty}\left(J_{\alpha}(s) J_{\alpha-1}(s)+J_{1-\alpha}(s) J_{-\alpha}(s)\right) d s,
\end{aligned}
$$

which implies the assertion.

Our next result will not be needed in the sequel, but it helps to clarify the behavior of $\rho_{\alpha}$ and demonstrates the methods which we shall use later on.

Lemma 2.3. Let $0<\alpha<1$. As $t \rightarrow \infty$,

$$
\rho_{\alpha}(t)=1-\frac{\sin \alpha \pi}{\pi} \frac{\cos 2 t}{t}+\mathcal{O}\left(t^{-2}\right) .
$$

Proof. The asymptotics AbSt, 9.2.5]

$$
J_{\nu}(t)=\sqrt{\frac{2}{\pi t}}\left(\cos \left(t-\frac{\pi}{4}-\frac{\nu \pi}{2}\right)-\frac{4 \nu^{2}-1}{8 t} \sin \left(t-\frac{\pi}{4}-\frac{\nu \pi}{2}\right)+\mathcal{O}\left(t^{-2}\right)\right),
$$

the formula (2.4) and elementary manipulations show that

$$
\rho_{\alpha}^{\prime}(t)=\frac{2}{\pi t}\left(\sin \alpha \pi \sin 2 t+\frac{(2 \alpha-1)^{2} \sin \alpha \pi}{4} \frac{\cos 2 t}{t}+\mathcal{O}\left(t^{-2}\right)\right) .
$$

Using that $\rho_{\alpha}(t)=1-\int_{t}^{\infty} \rho_{\alpha}^{\prime}(s) d s$ by Lemma 2.2 we obtain the assertion by repeated integration by parts.

\subsection{Moments of the spectral density}

For any $\gamma>-1$ let us define

$$
\sigma_{\alpha, \gamma}(r):=\int_{0}^{1}(1-\lambda)^{\gamma} \rho_{\alpha}(\sqrt{\lambda} r) d \lambda, \quad r \geq 0 .
$$

We are interested in the asymptotic behavior of this quantity or, more precisely, in the way it approaches its limit.

Theorem 2.4. Let $0<\alpha<1$ and $\gamma>-1$. As $r \rightarrow \infty$,

$$
\sigma_{\alpha, \gamma}(r)=\frac{1}{\gamma+1}-\Gamma(\gamma+1) \frac{\sin \alpha \pi}{\pi} \frac{\sin \left(2 r-\frac{1}{2} \gamma \pi\right)}{r^{2+\gamma}}+\mathcal{O}\left(r^{-3-\gamma}\right) .
$$

For the proof we note that by Lemma 2.2 and dominated convergence

In view of

$$
\lim _{r \rightarrow \infty} \sigma_{\alpha, \gamma}(r)=\int_{0}^{1}(1-\lambda)^{\gamma} d \lambda=\frac{1}{\gamma+1} .
$$

$$
\sigma_{\alpha, \gamma}(r)=\frac{1}{\gamma+1}-\int_{r}^{\infty} \sigma_{\alpha, \gamma}^{\prime}(s) d s,
$$

Theorem 2.4 follows via integration by parts from 
Proposition 2.5. Let $0<\alpha<1$ and $\gamma>-1$. As $r \rightarrow \infty$,

$$
\sigma_{\alpha, \gamma}^{\prime}(r)=\frac{\Gamma(\gamma+1)}{r^{\gamma+2}} \frac{\sin \alpha \pi}{\pi}\left(-2 \cos \left(2 r-\frac{\gamma \pi}{2}\right)-\frac{d_{1}}{r} \sin \left(2 r-\frac{\gamma \pi}{2}\right)+\mathcal{O}\left(r^{-2}\right)\right)
$$

where $d_{1}:=\frac{1}{8}\left(1-4\left(\left(\gamma+\frac{3}{2}\right)^{2}+(1-2 \alpha)^{2}\right)\right.$.

We defer the rather technical proof of this proposition to the following subsection. We would like to point out that (2.6) can be proved in an elementary way if $\alpha=\frac{1}{2}$ due to the formula (2.3). Indeed, if we simplify further by taking $\gamma=1$, then

$$
\begin{aligned}
\sigma_{1 / 2,1}^{\prime}(r) & =\frac{2}{\pi r} \int_{0}^{1}(1-\lambda) \sin (2 \sqrt{\lambda} r) d \lambda=\frac{4}{\pi r} \int_{0}^{1} k\left(1-k^{2}\right) \sin 2 k r d k \\
& =-\frac{2}{\pi r}\left(\frac{\sin 2 r}{r^{2}}+\frac{3 \cos 2 r}{2 r^{3}}+\mathcal{O}\left(r^{-4}\right)\right)
\end{aligned}
$$

by integration by parts. This is (2.6) in this special case.

\subsection{Proof of Proposition 2.5}

We shall need

Lemma 2.6. Let $\nu>-1 / 2$ and $\gamma>-1$. Then

$$
\int_{0}^{1} J_{\nu+1 / 2}(k r) J_{\nu-1 / 2}(k r) k^{2}\left(1-k^{2}\right)^{\gamma} d k=c_{\gamma} \int_{0}^{1} J_{2 \nu}(2 k r) k\left(1-k^{2}\right)^{\gamma+1 / 2} d k
$$

with

$$
c_{\gamma}:=\frac{1}{\sqrt{\pi}} \frac{\Gamma(\gamma+1)}{\Gamma\left(\gamma+\frac{3}{2}\right)} .
$$

Proof. By virtue of the series representations [AbSt, 9.1.10, 9.1.14] for $\alpha>0$,

$$
\begin{aligned}
J_{\alpha-1}(t) & =\left(\frac{t}{2}\right)^{\alpha-1} \sum_{n=0}^{\infty} \frac{(-1)^{n}}{n ! \Gamma(\alpha+n)}\left(\frac{t}{2}\right)^{2 n}, \\
J_{\alpha}(t) J_{\alpha-1}(t) & =\left(\frac{t}{2}\right)^{2 \alpha-1} \sum_{n=0}^{\infty} \frac{(-1)^{n} \Gamma(2 \alpha+2 n)}{n ! \Gamma(\alpha+n) \Gamma(\alpha+n+1) \Gamma(2 \alpha+n)}\left(\frac{t}{2}\right)^{2 n},
\end{aligned}
$$

the statement is equivalent to

$$
\begin{array}{r}
\left(\frac{r}{2}\right)^{2 \nu-1} \sum_{n=0}^{\infty} \frac{(-1)^{n}(r / 2)^{2 n} \Gamma(2 \nu+2 n)}{n ! \Gamma(2 \nu+n) \Gamma(\nu+n) \Gamma(\nu+n+1)} \int_{0}^{1} k^{2 \nu+2 n+1}\left(1-k^{2}\right)^{\gamma} d k \\
=\frac{r^{2 \nu-1}}{\sqrt{\pi}} \frac{\Gamma(\gamma+1)}{\Gamma\left(\gamma+\frac{3}{2}\right)} \sum_{n=0}^{\infty} \frac{(-1)^{n} r^{2 n}}{n ! \Gamma(2 \nu+n)} \int_{0}^{1} k^{2 \nu+2 n}\left(1-k^{2}\right)^{\gamma+1 / 2} d k,
\end{array}
$$


an equality which actually holds termwise. This follows by the beta function identity

$$
2 \int_{0}^{1} k^{2 \alpha-1}\left(1-k^{2}\right)^{\beta-1} d k=\int_{0}^{1} t^{\alpha-1}(1-t)^{\beta-1} d t=B(\alpha, \beta)=\frac{\Gamma(\alpha) \Gamma(\beta)}{\Gamma(\alpha+\beta)}
$$

and the duplication formula $\sqrt{\pi} \Gamma(2 \alpha)=2^{2 \alpha-1} \Gamma(\alpha) \Gamma\left(\alpha+\frac{1}{2}\right)$ [AbSt, 6.1.18].

Lemma 2.7. Let $\beta>0$ and $-2<\nu<2$. The following decomposition holds:

$$
\int_{0}^{1} J_{\nu}(k r) k\left(1-k^{2}\right)^{\beta-1} d k=f_{\beta, \nu}(r)+g_{\beta, \nu}(r),
$$

where $g_{\beta,-\nu}(r)=-g_{\beta, \nu}(r)$ and

$$
f_{\beta, \nu}(r)=\frac{\Gamma(\beta)}{2 \sqrt{\pi}}\left(\frac{2}{r}\right)^{\beta+1 / 2}\left[\cos \left(r-r_{0}\right)+\frac{d_{1}}{r} \sin \left(r-r_{0}\right)+\mathcal{O}\left(r^{-2}\right)\right], \quad r \rightarrow \infty,
$$

with

$$
r_{0}:=\frac{\pi}{2}\left(\beta+\nu+\frac{1}{2}\right) \quad \text { and } \quad d_{1}:=\frac{1}{8}-\frac{\beta^{2}+\nu^{2}}{2} .
$$

The function $g_{\beta, \nu}$ has a power-like, non-oscillatory behavior at infinity and dominates $f_{\beta, \nu}$ when $\beta>\frac{3}{2}$. Its odd parity with respect to $\nu$ is, however, the property vital to us, since it leads to a useful cancellation.

Proof. The left-hand side in (2.9) can be expressed as a generalized hypergeometric function. Recall that for $p, q \in \mathbb{N}_{0}, p \leq q$ and $\alpha_{1}, \ldots, \alpha_{p}, \beta_{1}, \ldots, \beta_{q} \in \mathbb{R} \backslash\left(-\mathbb{N}_{0}\right)$,

$$
{ }_{p} F_{q}\left(\begin{array}{c}
\alpha_{1}, \alpha_{2}, \ldots, \alpha_{p} \\
\beta_{1}, \beta_{2}, \ldots, \beta_{q}
\end{array} \mid z\right)=\sum_{n=0}^{\infty} \frac{\left(\alpha_{1}\right)_{n}\left(\alpha_{2}\right)_{n} \cdots\left(\alpha_{p}\right)_{n}}{\left(\beta_{1}\right)_{n}\left(\beta_{2}\right)_{n} \cdots\left(\beta_{q}\right)_{n}} \frac{z^{n}}{n !}, \quad z \in \mathbb{C}
$$

where $(\alpha)_{n}=\Gamma(\alpha+n) / \Gamma(\alpha)=\alpha(\alpha+1) \cdots(\alpha+n-1)$. By [Lu, 13.3.2(10)],

$$
\begin{aligned}
& \int_{0}^{1} J_{\nu}(k r) k\left(1-k^{2}\right)^{\beta-1} d k=\int_{0}^{\pi / 2} J_{\nu}(r \sin \theta) \cos ^{2 \beta-1} \theta \sin \theta d \theta \\
= & \frac{r^{\nu} \Gamma(\beta) \Gamma(\nu / 2+1)}{2^{\nu+1} \Gamma(\nu+1) \Gamma(\beta+\nu / 2+1)}{ }_{1} F_{2}\left(\begin{array}{c}
\nu / 2+1 \\
\nu+1, \beta+\nu / 2+1
\end{array} \mid-\frac{r^{2}}{4}\right) .
\end{aligned}
$$

Next we use the asymptotics of the generalized hypergeometric function $\mathrm{Lu}$, 1.3.3 $(5,7,8,13)]$,

$$
\begin{aligned}
& \frac{\Gamma(\nu / 2+1)}{\Gamma(\nu+1) \Gamma(\beta+\nu / 2+1)}{ }_{1} F_{2}\left(\begin{array}{c|c}
\nu / 2+1 \\
\nu+1, \beta+\nu / 2+1
\end{array} \mid-\frac{r^{2}}{4}\right) \\
& \sim \frac{(2 / r)^{\beta+\nu+1 / 2}}{2 \sqrt{\pi}}\left[e^{i\left(r-r_{0}\right)} \sum_{n=0}^{\infty} d_{n}(i r)^{-n}+e^{-i\left(r-r_{0}\right)} \sum_{n=0}^{\infty} d_{n}(-i r)^{-n}\right] \\
& +\frac{\nu(2 / r)^{\nu+2}}{2 \Gamma(\beta)}{ }_{3} F_{0}\left(\begin{array}{c|c}
1+\nu / 2,1-\nu / 2,1-\beta & -\frac{4}{r^{2}}
\end{array}\right), \quad r \rightarrow \infty,
\end{aligned}
$$


with $r_{0}, d_{1}$ as in (2.10) and $d_{0}=1$. We emphasize that the $\sim$ sign means equality in the sense of asymptotic expansions, and that ${ }_{3} F_{0}$ is not a well-defined function but denotes an asymptotic expansion. We define $\nu^{-1} r^{2} g_{\beta, \nu}(r)$ as a finite approximation to ${ }_{3} F_{0}$. Namely, let $K$ denote the smallest non-negative integer such that $2 K \geq$ $\beta-\frac{3}{2}$ and put

$$
g_{\beta, \nu}(r):=\frac{\nu}{r^{2}} \sum_{n=0}^{K}(1+\nu / 2)_{n}(1-\nu / 2)_{n}(1-\beta)_{n} \frac{(-1)^{n}}{n !}\left(\frac{2}{r}\right)^{2 n} .
$$

This function is antisymmetric in $\nu$ and

$$
\begin{aligned}
f_{\beta, \nu}(r):= & \int_{0}^{1} J_{\nu}(k r) k\left(1-k^{2}\right)^{\beta-1} d k-g_{\beta, \nu}(r) \\
= & \frac{r^{\nu} \Gamma(\beta)}{2^{\nu+1}} \frac{(2 / r)^{\beta+\nu+1 / 2}}{2 \sqrt{\pi}}\left[e^{i\left(r-r_{0}\right)}\left(d_{0}+\frac{d_{1}}{i r}\right)+e^{-i\left(r-r_{0}\right)}\left(d_{0}-\frac{d_{1}}{i r}\right)\right. \\
& \left.\quad+\mathcal{O}\left(r^{-2}\right)\right]+\mathcal{O}\left(r^{-2(K+2)}\right) \\
= & \frac{\Gamma(\beta)}{2 \sqrt{\pi}}\left(\frac{2}{r}\right)^{\beta+1 / 2}\left[d_{0} \cos \left(r-r_{0}\right)+\frac{d_{1}}{r} \sin \left(r-r_{0}\right)+\mathcal{O}\left(r^{-2}\right)\right]
\end{aligned}
$$

as claimed.

Now everything is in place for the

Proof of Proposition 2.5. By Lemmas 2.2 and 2.6.

$$
\begin{aligned}
\sigma_{\alpha, \gamma}^{\prime}(r) & =2 \int_{0}^{1} k^{2}\left(1-k^{2}\right)^{\gamma} \rho_{\alpha}^{\prime}(k r) d k \\
& =2 \int_{0}^{1}\left(J_{\alpha}(k r) J_{\alpha-1}(k r)+J_{1-\alpha}(k r) J_{-\alpha}(k r)\right) k^{2}\left(1-k^{2}\right)^{\gamma} d k \\
& =2 c_{\gamma} \int_{0}^{1}\left(J_{2 \alpha-1}(2 k r)+J_{1-2 \alpha}(2 k r)\right) k\left(1-k^{2}\right)^{\gamma+1 / 2} d k
\end{aligned}
$$

with $c_{\gamma}$ from (2.7). Now we apply Lemma 2.7 with $\beta=\gamma+\frac{3}{2}$ and $\nu= \pm(2 \alpha-1)$. Using the antisymmetry of $g_{\beta, \nu}$ we find that

$$
\sigma_{\alpha, \gamma}^{\prime}(r)=2 c_{\gamma}\left(f_{\gamma+3 / 2,2 \alpha-1}(r)+f_{\gamma+3 / 2,1-2 \alpha}(r)\right)
$$

and the assertion follows after elementary manipulations from the asymptotics of $f_{\beta, \nu}$ given in Lemma 2.7 .

\subsection{The Laplace transform of the spectral density}

In this subsection we are interested in the quantity

$$
\sigma_{\alpha, \infty}(r):=\int_{0}^{\infty} e^{-\lambda} \rho_{\alpha}(\sqrt{\lambda} r) d \lambda, \quad r \geq 0 .
$$

Note that this is essentially the Laplace transform of the function $\rho_{\alpha}(\sqrt{\bullet})$. 
Theorem 2.8. For all $0<\alpha<1$ the function $\sigma_{\alpha, \infty}$ is strictly increasing from 0 to 1 on $[0, \infty)$. In particular, $\sigma_{\alpha, \infty}(r)<1$ for all $r \geq 0$.

This theorem shows that the oscillations we observed for $\sigma_{\alpha, \gamma}$ are no longer present.

Proof. Since $\rho_{\alpha}=\rho_{1-\alpha}$ and hence $\sigma_{\alpha, \infty}=\sigma_{1-\alpha, \infty}$, it suffices to treat the case $0<\alpha \leq 1 / 2$. By the properties of $\rho_{\alpha}$ (see Lemma 2.2) and dominated convergence we get $\sigma_{\alpha, \infty}(0)=0$ and $\sigma_{\alpha, \infty}(r) \rightarrow 1$ as $r \rightarrow \infty$. Again by Lemma 2.2 ,

$$
\begin{aligned}
\sigma_{\alpha, \infty}^{\prime}(r) & =2 \int_{0}^{\infty} k^{2} e^{-k^{2}} \rho_{\alpha}^{\prime}(k r) d k \\
& =2 \int_{0}^{\infty} k^{2} e^{-k^{2}}\left(J_{\alpha}(k r) J_{\alpha-1}(k r)+J_{1-\alpha}(k r) J_{-\alpha}(k r)\right) d k
\end{aligned}
$$

and hence according to [Lu, 13.4.1(10)],

$$
\begin{aligned}
\sigma_{\alpha, \infty}^{\prime}(r)=r^{1-2 \alpha}\left[\frac{2^{1-2 \alpha}}{r^{2(1-2 \alpha)} \Gamma(\alpha)}{ }_{1} F_{1}\right. & \left(\begin{array}{c}
\alpha+\frac{1}{2} \\
2 \alpha
\end{array} \mid-r^{2}\right) \\
& \left.+\frac{1}{2^{1-2 \alpha} \Gamma(1-\alpha)}{ }_{1} F_{1}\left(\begin{array}{c}
\frac{3}{2}-\alpha \\
2-2 \alpha
\end{array} \mid-r^{2}\right)\right] .
\end{aligned}
$$

In the special case $\alpha=1 / 2$ we note that

$$
\sigma_{1 / 2, \infty}^{\prime}(r)=\frac{2}{\sqrt{\pi}}{ }_{1} F_{1}\left(\begin{array}{c|c}
1 \\
1
\end{array} \mid-r^{2}\right)=\frac{2}{\sqrt{\pi}} e^{-r^{2}} .
$$

If $0<\alpha<\frac{1}{2}$ we can apply the Kummer transformations [AbSt, 13.1.27] (note that ${ }_{1} F_{1}(a, b ; z)=M(a, b, z)$ in $\left.[\mathrm{AbSt}]\right)$ to get

$$
\begin{aligned}
\sigma_{\alpha, \infty}^{\prime}(r)=r^{1-2 \alpha} e^{-r^{2}}\left[\frac{2^{1-2 \alpha}}{r^{2(1-2 \alpha)} \Gamma(\alpha)}{ }_{1}\right. & F_{1}\left(\begin{array}{c}
\alpha-\frac{1}{2} \\
2 \alpha
\end{array} \mid r^{2}\right) \\
& \left.+\frac{1}{2^{1-2 \alpha} \Gamma(1-\alpha)}{ }_{1} F_{1}\left(\begin{array}{c}
\frac{1}{2}-\alpha \\
2-2 \alpha
\end{array}\right) r^{2}\right) .
\end{aligned}
$$

By elementary properties of the gamma function this can be rewritten as

$$
\sigma_{\alpha, \infty}^{\prime}(r)=\frac{2 \sin \alpha \pi}{\sqrt{\pi}} r^{1-2 \alpha} e^{-r^{2}} U\left(\frac{1}{2}-\alpha, 2-2 \alpha, r^{2}\right)
$$

where $\mathrm{AbSt}, 13.1 .3$

$$
\begin{aligned}
U\left(\frac{1}{2}-\alpha, 2-2 \alpha, r^{2}\right)=\frac{\pi}{\sin 2 \alpha \pi} & \frac{1}{r^{2(1-2 \alpha)}}\left[\frac{1}{\Gamma\left(\frac{1}{2}-\alpha\right) \Gamma(2 \alpha)}{ }_{1} F_{1}\left(\begin{array}{c}
\alpha-\frac{1}{2} \\
2 \alpha
\end{array} \mid r^{2}\right)\right. \\
& \left.-\frac{r^{2(1-2 \alpha)}}{\Gamma\left(\alpha-\frac{1}{2}\right) \Gamma(2-2 \alpha)}{ }_{1} F_{1}\left(\begin{array}{c}
\frac{1}{2}-\alpha \\
2-2 \alpha
\end{array} \mid r^{2}\right)\right] .
\end{aligned}
$$

$U$ is positive by the integral representation [AbSt, 13.2.5], and this proves the theorem. 


\section{Counterexample to the generalized diamagnetic inequality}

Following [ELoV] we consider the question: Which non-negative convex functions $\varphi$ vanishing at infinity satisfy

$$
\operatorname{tr} \chi_{\Omega} \varphi\left(H_{\alpha}\right) \chi_{\Omega} \leq \operatorname{tr} \chi_{\Omega} \varphi(-\Delta) \chi_{\Omega} \quad \text { for all bounded domains } \Omega \subset \mathbb{R}^{2} \text { ? }
$$

By (2.1) the statement (3.1) is equivalent to the pointwise inequality

$$
\int_{0}^{\infty} \varphi(\lambda) \rho_{\alpha}(\sqrt{\lambda} r) d \lambda \leq \int_{0}^{\infty} \varphi(\lambda) d \lambda, \quad \text { for all } r \in[0, \infty) .
$$

Note that (3.1) is true for the family of functions $\varphi(\lambda)=e^{-t \lambda}, t>0$; we shall prove this (even with strict inequality) in Remark 3.2 below. Alternatively, it follows from the 'ordinary' diamagnetic inequality (see, e.g., $\mathrm{HuS}$ ),

$$
\left|\exp \left(-t H_{\alpha}\right) u\right| \leq \exp (-t(-\Delta))|u| \text { a.e., } \quad u \in L_{2}\left(\mathbb{R}^{2}\right),
$$

and [S, Thm. 2.13], since

$$
\left\|\chi_{\Omega} \exp \left(-\frac{t}{2} H_{\alpha}\right)\right\|_{2}^{2}=\operatorname{tr} \chi_{\Omega} \exp \left(-t H_{\alpha}\right) \chi_{\Omega} .
$$

We point out that the validity of (3.1) for $\varphi(\lambda)=e^{-t \lambda}, t>0$, actually implies that it holds for any function of the form

$$
\varphi(\lambda)=\int_{0}^{\infty} e^{-t \lambda} w(t) d t, \quad w \geq 0
$$

In connection with a Berezin-Li-Yau-type inequality one is particularly interested in the functions

$$
\varphi(\lambda)=(\lambda-\Lambda)_{-}^{\gamma}, \quad \gamma \geq 1, \Lambda>0 .
$$

Note that these functions cannot be expressed in the form (3.3).

Theorem 3.1. Let $0<\alpha<1$ and let $\varphi$ be given by (3.4) for some $\gamma \geq 1, \Lambda>0$. Then the generalized diamagnetic inequality (3.1) is violated. More precisely, there exist constants $C_{1}, C_{2}>0$ (depending on $\alpha$ and $\gamma$, but not on $\Lambda$ ) such that for all $|x| \geq C_{1} \Lambda^{-1 / 2}$,

$$
\left|\varphi\left(H_{\alpha}\right)(x, x)-\varphi(-\Delta)(x, x)+A_{\alpha, \gamma}(\Lambda) \frac{\sin \left(2 \sqrt{\Lambda}|x|-\frac{1}{2} \gamma \pi\right)}{|x|^{2+\gamma}}\right| \leq C_{2} \frac{\Lambda^{(\gamma-1) / 2}}{|x|^{3+\gamma}}
$$

with $A_{\alpha, \gamma}(\Lambda):=(2 \pi)^{-2} \Lambda^{\gamma / 2} \Gamma(\gamma+1) \sin \alpha \pi$.

Proof. By 2.1) and the scaling $\lambda \mapsto \Lambda \lambda$,

$$
\varphi\left(H_{\alpha}\right)(x, x)=\frac{\Lambda^{\gamma+1}}{4 \pi} \int_{0}^{1}(1-\lambda)^{\gamma} \rho_{\alpha}(\sqrt{\Lambda \lambda}|x|) d \lambda=\frac{\Lambda^{\gamma+1}}{4 \pi} \sigma_{\alpha, \gamma}(\sqrt{\Lambda}|x|),
$$

where we used the notation (2.5). Note that $\varphi(-\Delta)(x, x)=(4 \pi(\gamma+1))^{-1} \Lambda^{\gamma+1}$. The expansion (3.5) is thus a consequence of Theorem 2.4. To prove that the generalized diamagnetic inequality (3.1) is violated, one can consider the domains 
$\Omega_{n}:=\left\{x \in \mathbb{R}^{2}:|\sqrt{\Lambda}| x\left|-r_{n}\right|<\varepsilon\right\}, n \in \mathbb{N}$, with $r_{n}:=\pi\left(n+\frac{1}{4}(\gamma-1)\right)$ and sufficiently small but fixed $\varepsilon>0$. It follows easily from (3.5) that (3.1) is violated for all large $n$.

Remark 3.2. The analogous statement (with the same proof) is valid for $-1<$ $\gamma<1$. Moreover, for exponential functions Theorem 2.8 implies that

$$
\exp \left(-t H_{\alpha}\right)(x, x)=\frac{1}{4 \pi t} \int_{0}^{\infty} \rho_{\alpha}\left(\sqrt{\lambda} \frac{|x|}{\sqrt{t}}\right) e^{-\lambda} d \lambda=\frac{1}{4 \pi t} \sigma_{\alpha, \infty}\left(\frac{|x|}{\sqrt{t}}\right)
$$

is strictly less than $(4 \pi t)^{-1}=\exp (-t(-\Delta))(x, x)$.

The following substitute for (3.1) will be useful later on.

Proposition 3.3. Let $0<\alpha<1$ and let $\varphi$ be given by (3.4) for some $\gamma>-1$, $\Lambda>0$. Then for all open sets $\Omega \subset \mathbb{R}^{2}$

$$
\operatorname{tr} \chi_{\Omega} \varphi\left(H_{\alpha}\right) \chi_{\Omega} \leq R_{\gamma}(\alpha) \operatorname{tr} \chi_{\Omega} \varphi(-\Delta) \chi_{\Omega}
$$

with

$$
R_{\gamma}(\alpha):=(\gamma+1) \sup _{r \geq 0} \int_{0}^{1}(1-\lambda)^{\gamma} \rho_{\alpha}(\sqrt{\lambda} r) d \lambda .
$$

Indeed, this is an immediate consequence of (3.6). This shows as well that the right-hand side of (3.8) yields the sharp constant in (3.7).

Remark 3.4. The constant $R_{\gamma}(\alpha)$ is strictly decreasing with respect to $\gamma$. Indeed, following AL we write, for $\gamma>\gamma^{\prime}>-1$ and $0 \leq \lambda \leq 1$,

$$
B\left(\gamma-\gamma^{\prime}, \gamma^{\prime}+1\right)(1-\lambda)^{\gamma}=\int_{0}^{1-\lambda}(1-\lambda-\mu)^{\gamma^{\prime}} \mu^{\gamma-\gamma^{\prime}-1} d \mu
$$

and find, for any $r \geq 0$,

$$
\begin{aligned}
& B\left(\gamma-\gamma^{\prime}, \gamma^{\prime}+1\right) \int_{0}^{1}(1-\lambda)^{\gamma} \rho_{\alpha}(\sqrt{\lambda} r) d \lambda \\
= & \int_{0}^{1}\left(\int_{0}^{1-\mu}(1-\lambda-\mu)^{\gamma^{\prime}} \rho_{\alpha}(\sqrt{\lambda} r) d \lambda\right) \mu^{\gamma-\gamma^{\prime}-1} d \mu \\
= & \int_{0}^{1} w(r \sqrt{1-\mu})(1-\mu)^{\gamma^{\prime}+1} \mu^{\gamma-\gamma^{\prime}-1} d \mu,
\end{aligned}
$$

where $w(s):=\int_{0}^{1}(1-\lambda)^{\gamma^{\prime}} \rho_{\alpha}(\sqrt{\lambda} s) d \lambda$. Since $w(s) \leq R_{\gamma^{\prime}}(\alpha)\left(\gamma^{\prime}+1\right)^{-1}$ one concludes that

$$
\begin{aligned}
R_{\gamma}(\alpha) & \leq R_{\gamma^{\prime}}(\alpha)\left(\gamma^{\prime}+1\right)^{-1}(\gamma+1) B\left(\gamma-\gamma^{\prime}, \gamma^{\prime}+1\right)^{-1} \int_{0}^{1}(1-\mu)^{\gamma^{\prime}+1} \mu^{\gamma-\gamma^{\prime}-1} d \mu \\
& =R_{\gamma^{\prime}}(\alpha)\left(\gamma^{\prime}+1\right)^{-1}(\gamma+1) B\left(\gamma-\gamma^{\prime}, \gamma^{\prime}+1\right)^{-1} B\left(\gamma-\gamma^{\prime}, \gamma^{\prime}+2\right)=R_{\gamma^{\prime}}(\alpha) .
\end{aligned}
$$

That this inequality is actually strict, follows from the fact that the supremum in (3.8) is attained for some $r_{0} \in(0, \infty)$ (see Theorem 2.4) and that $w\left(r_{0} \sqrt{1-\mu}\right.$ ) is non-constant with respect to $\mu$. 
We close this section by giving numerical values for $R_{\gamma}(\alpha)$. Note that

$$
\begin{aligned}
R_{\gamma}(\alpha) & =2(\gamma+1) \sup _{r \geq 0} \int_{0}^{1}\left(1-k^{2}\right)^{\gamma} \rho_{\alpha}(k r) k d k \\
& =\sup _{r \geq 0} r \int_{0}^{1}\left(1-k^{2}\right)^{\gamma+1} \rho_{\alpha}^{\prime}(k r) d k \\
& =\sup _{r \geq 0} r \int_{0}^{1}\left(1-k^{2}\right)^{\gamma+1}\left(J_{\alpha}(k r) J_{\alpha-1}(k r)+J_{1-\alpha}(k r) J_{-\alpha}(k r)\right) d k .
\end{aligned}
$$

The integral can be evaluated by some quadrature algorithm and by Theorem 2.4 the supremum is attained for finite $r$. This allows us to compute approximate values of $R_{\gamma}(\alpha)\left(=R_{\gamma}(1-\alpha)\right)$,

\begin{tabular}{l|lllll} 
& $R_{\gamma}(0.1)$ & $R_{\gamma}(0.2)$ & $R_{\gamma}(0.3)$ & $R_{\gamma}(0.4)$ & $R_{\gamma}(0.5)$ \\
\hline$\gamma=0$ & 1.01682 & 1.03262 & 1.04422 & 1.05151 & 1.05397 \\
$\gamma=\frac{1}{2}$ & 1.01027 & 1.02050 & 1.02781 & 1.03241 & 1.03395 \\
$\gamma=1$ & 1.00650 & 1.01351 & 1.01833 & 1.02138 & 1.02238 \\
$\gamma=\frac{3}{2}$ & 1.00417 & 1.00920 & 1.01250 & 1.01457 & 1.01524 \\
$\gamma=2$ & 1.00267 & 1.00642 & 1.00874 & 1.01019 & 1.01065
\end{tabular}

In addition to the monotonicity in $\gamma$ (see Remark 3.4) the constant $R_{\gamma}(\alpha)$ seems to be strictly increasing in $\alpha$, but we have not been able to prove this.

\section{A magnetic Berezin-Li-Yau inequality}

As in the introduction, let $\Omega \subset \mathbb{R}^{2}$ be a bounded domain and define $H_{\alpha}^{\Omega}$ in $L_{2}(\Omega)$ through the closure of the quadratic form $\left\|\left(\mathbf{D}-\alpha \mathbf{A}_{0}\right) u\right\|^{2}$ on $C_{0}^{\infty}(\Omega \backslash\{0\})$. For eigenvalue moments of this operator we shall prove

Theorem 4.1. Let $0<\alpha<1, \gamma \geq 1$ and $\Omega \subset \mathbb{R}^{2}$ be a bounded domain such that the operator $H_{\alpha}^{\Omega}$ has discrete spectrum. Then for any $\Lambda>0$,

$$
\operatorname{tr}\left(H_{\alpha}^{\Omega}-\Lambda\right)_{-}^{\gamma} \leq R_{\gamma}(\alpha) \frac{1}{4 \pi(\gamma+1)}|\Omega| \Lambda^{\gamma+1}
$$

with the constant $R_{\gamma}(\alpha)$ from (3.8).

As explained in the introduction,

$$
\frac{1}{4 \pi(\gamma+1)}|\Omega| \Lambda^{\gamma+1}=\frac{1}{(2 \pi)^{2}} \iint_{\mathbb{R}^{2} \times \Omega}\left(|\xi|^{2}-\Lambda\right)_{-}^{\gamma} d \xi d x
$$

is the semi-classical approximation for $\operatorname{tr}\left(H_{\alpha}^{\Omega}-\Lambda\right)_{-}^{\gamma}$. Unfortunately, we can only prove (4.1) with an excess factor $R_{\gamma}(\alpha)$, which is strictly larger than one by Theorem 2.4. Based on numerical calculations (see next section) we conjecture that (4.1) should be valid with $R_{\gamma}(\alpha)=1$. 
Proof. We first note that

$$
\operatorname{tr}\left(H_{\alpha}^{\Omega}-\Lambda\right)_{-} \leq \operatorname{tr} \chi_{\Omega}\left(\chi_{\Omega} H_{\alpha} \chi_{\Omega}-\Lambda\right)_{-} \chi_{\Omega}
$$

Indeed, let $\left(\omega_{j}\right)$ be an orthonormal basis of eigenfunctions of $H_{\alpha}^{\Omega}$. Then the extension $\tilde{\omega}_{j}$ of $\omega_{j}$ by zero belongs to the form domain of $\chi_{\Omega} H_{\alpha} \chi_{\Omega}$ and

$$
\begin{aligned}
\operatorname{tr}\left(H_{\alpha}^{\Omega}-\Lambda\right)_{-} & =\sum_{j}\left(\left(H_{\alpha}^{\Omega}-\Lambda\right) \omega_{j}, \omega_{j}\right)_{-}=\sum_{j}\left(\left(\chi_{\Omega} H_{\alpha} \chi_{\Omega}-\Lambda\right) \tilde{\omega}_{j}, \tilde{\omega}_{j}\right)_{-} \\
& \leq \sum_{j}\left(\left(\chi_{\Omega} H_{\alpha} \chi_{\Omega}-\Lambda\right)_{-} \tilde{\omega}_{j}, \tilde{\omega}_{j}\right)=\operatorname{tr} \chi_{\Omega}\left(\chi_{\Omega} H_{\alpha} \chi_{\Omega}-\Lambda\right)_{-} \chi_{\Omega} .
\end{aligned}
$$

Now let $\varphi$ be a convex function of the form $\varphi(\lambda)=\int(\lambda-\mu)_{-} w(\mu) d \mu$ for some $w \geq 0$. Then it follows from (4.2) that

$$
\operatorname{tr} \varphi\left(H_{\alpha}^{\Omega}\right) \leq \operatorname{tr} \chi_{\Omega} \varphi\left(\chi_{\Omega} H_{\alpha} \chi_{\Omega}\right) \chi_{\Omega},
$$

and hence by the Berezin-Lieb inequality (see [B2], L] and also [LaSa], La]),

$$
\operatorname{tr} \varphi\left(H_{\alpha}^{\Omega}\right) \leq \operatorname{tr} \chi_{\Omega} \varphi\left(\chi_{\Omega} H_{\alpha} \chi_{\Omega}\right) \chi_{\Omega} \leq \operatorname{tr} \chi_{\Omega} \varphi\left(H_{\alpha}\right) \chi_{\Omega} .
$$

As already noted in Remark 3.4, the function $\varphi(\lambda)=(\lambda-\Lambda)_{-}^{\gamma}$ is of the considered form. Noting that in this case $\operatorname{tr} \chi_{\Omega} \varphi(-\Delta) \chi_{\Omega}=(4 \pi(\gamma+1))^{-1}|\Omega| \Lambda^{\gamma+1}$, the assertion follows from Proposition 3.3 .

\section{Numerical experiments}

\subsection{Numerical evaluation of the magnetic Berezin-Li-Yau constant}

In this section we present a numerical study, somewhat in the spirit of LT, Appendix $\mathrm{A}$ ], of the constant $R_{\gamma}(\alpha)$ appearing in (4.1) for $H_{\alpha}^{\Omega}$ on various domains. We consider the unit disc, a square and three annuli:

$$
\begin{array}{ll}
A:=\{x:|x|<1\}, & C:=\{x: 1<|x|<1.1\}, \\
B:=\left\{x: \max \left(\left|x_{1}\right|,\left|x_{2}\right|\right)<1\right\}, & D:=\{x: 1<|x|<2\}, \\
& E:=\{x: 1<|x|<11\} .
\end{array}
$$

To expose possible diamagnetic effects we perform identical experiments for $\alpha=0$ and $\alpha=0.2$ throughout. If the eigenvalues $\lambda_{1}, \lambda_{2}, \ldots$ are known, the task is to determine

$$
R_{\gamma}=\sup _{\Lambda} r_{\gamma}(\Lambda), \quad \text { where } r_{\gamma}(\Lambda):=\frac{4 \pi(\gamma+1)}{|\Omega| \Lambda^{\gamma+1}} \sum_{j: \lambda_{j}<\Lambda}\left(\Lambda-\lambda_{j}\right)^{\gamma} .
$$

Since the sum is simply the counting function if $\gamma=0, r_{0}$ is decreasing for all $\Lambda$ but the eigenvalues. In the physically important case $\gamma=1$, the quotient $r_{1}$ is continuous but $r_{1}^{\prime}$ has jump increases at the eigenvalues. Figures 1 and 22 show, respectively, the schematic behaviour of $r_{\gamma}$ near the bottom of the spectrum and for large $\Lambda$ in the case where $R_{\gamma}=1$ (in fact, the spectrum of $H_{0}^{B}$ has been considered). Figure 3 depicts $r_{0}(\Lambda)$ for an operator with $R_{\gamma}>1$ (see [H, Thm. 3.2]), namely 
the Schrödinger operator $H_{\alpha}-|x|^{-1}$. The $\times$ symbols on the $x$ axis indicate the eigenvalue loci.

\subsection{Computation of the eigenvalues}

By separation of variables the spectrum of $H_{\alpha}^{A}$ is the union of the spectra of the family of one-dimensional problems

$$
-u^{\prime \prime}+\frac{(n-\alpha)^{2}-1 / 4}{r^{2}} u=\lambda u, \quad u(1)=0, \quad u \in L^{2}(0,1),
$$

parametrized by $n \in \mathbb{Z} . \sqrt{r} J_{|n-\alpha|}(\sqrt{\lambda} r)$ is an eigenfunction provided $\lambda$ is chosen to that $J_{|n-\alpha|}(\sqrt{\lambda})=0$, i.e., the eigenvalues are squares of the zeros of the Bessel function of the first kind. Using standard numerical procedures for this task we compute all eigenvalues below 10,000 (approximately 2,500) of $H_{0}^{A}$ and all eigenvalues below 50,000 (approx. 12,000) of $H_{0.2}^{A}$. The lowest eigenvalues of the two operators can be viewed in Figure 4. The reader interested in the dependence of the eigenvalues on the parameter $\alpha$ may find Figure 6 useful, where the lowest eigenvalues of $H_{\alpha}^{A}$ as functions of $\alpha \in\left[0, \frac{1}{2}\right]$ are shown. We recall that $H_{\alpha}^{A}$ and $H_{1-\alpha}^{A}$ share the same spectrum.

Similarly, the eigenvalue problems for $H_{\alpha}^{C}, H_{\alpha}^{D}$ and $H_{\alpha}^{E}$ are reduced to

$$
-u^{\prime \prime}+\frac{(n-\alpha)^{2}-1 / 4}{r^{2}} u=\lambda u, \quad u\left(r_{1}\right)=0=u\left(r_{2}\right), \quad u \in L^{2}\left(r_{1}, r_{2}\right) .
$$

In order that the general solution $\sqrt{r}\left(c_{1} J_{|n-\alpha|}(\sqrt{\lambda} r)+c_{2} Y_{|n-\alpha|}(\sqrt{\lambda} r)\right)$ satisfy the Dirichlet conditions we need to fix the ratio $c_{1} / c_{2}$ and take $\lambda$ such that

$$
J_{|n-\alpha|}\left(\sqrt{\lambda} r_{1}\right) Y_{|n-\alpha|}\left(\sqrt{\lambda} r_{2}\right)=J_{|n-\alpha|}\left(\sqrt{\lambda} r_{2}\right) Y_{|n-\alpha|}\left(\sqrt{\lambda} r_{1}\right) .
$$

Equivalently, $\lambda=x^{2} / r_{1}^{2}$ is an eigenvalue whenever $x$ is a zero of the cross-product $J_{\nu}(t) Y_{\nu}(\mu t)-J_{\nu}(\mu t) Y_{\nu}(t)$ for $\mu=r_{2} / r_{1}, \nu=|n-\alpha|$. Since the amplitude of the oscillation grows approximately as $\mu^{\nu} / \nu$, the computation of the zeros is nontrivial for large orders. By searching in the negative $t$ direction and making use of the simple fact that the asymptotic spacing of the zeros is $\pi /(\mu-1)$ AbSt, 9.5.28], we circumvent much of these difficulties in comparison to routines such as MATHEMATICA's BesselJYJYZeros. This allows us to compute, for $\alpha=0$ and 0.2 , all eigenvalues below 10,000 (approx. 400) of $H_{\alpha}^{C}$, all eigenvalues below 1,000 (approx. 550) of $H_{\alpha}^{D}$ and all eigenvalues below 300 (approx. 9,000) of $H_{\alpha}^{E}$.

$H_{0}^{B}$ is simply the Dirichlet Laplacian on $[-1,1]^{2}$, so for all $k, l \in \mathbb{N}$,

$$
\sin \left(k \pi \frac{x+1}{2}\right) \sin \left(l \pi \frac{y+1}{2}\right)
$$

is an eigenfunction and $\frac{\pi^{2}}{4}\left(k^{2}+l^{2}\right)$ an eigenvalue. For $\alpha \neq 0$ the absence of radial symmetry prevents us from solving the eigenvalue problem for $H_{\alpha}^{B}$ exactly. We therefore resort to the finite element method as implemented in Comsol MuLTIPHYSICS to compute approximate eigenvalues. A 8,800-element mesh, gradually refined near the singularity, is used to compute the 250 lowest eigenvalues, all less 
than 840. Providing an a priori bound on the computational error would be beyond the scope of this article; it is an encouraging fact, however, that the approximate eigenvalues of $H_{0.2}^{A}$ do not differ from the exact ones by more than $0.8 \%$. Figure 5 is the analogue of Figure 4 for $H_{\alpha}^{B}$.

\subsection{Outcome of the experiments}

We are obviously constrained to computing (5.1) with $\Lambda$ restricted to a finite interval but provided this interval is sufficiently large it should be possible to tell the behaviors depicted in Figures 2 and 3 apart. Particularly if the function $r_{\gamma}$ tends steadily to 1 from below we can conclude with certainty that its supremum will eventually be this number in virtue of the eigenvalue asymptotics. It turns out that all the operators we consider, whether $\alpha=0$ or 0.2 , seem to obey a semi-classical eigenvalue estimate with unit constant for both $\gamma=0$ and 1 .

What however differs is the rate of convergence to 1 , e.g., $r_{\gamma}$ increases slower for $H_{\alpha}^{C}$ than for $H_{\alpha}^{E}$ since $|C|<|E|$, implying that the point spectrum of the former operator is sparser. To see the influence of $\alpha$ on $r_{\gamma}$ we invite the reader to compare Figures 7, 8 and 9. Note that $r_{\gamma}$ has the most irregular behavior in the cases $\alpha=0$ and $\alpha=0.5$ due to the high degree of degeneracy of the eigenvalues, cf. Figure 6, Moreover we have the impression, from looking at Figures 1, 7 and 10, that the shape of the domain has a limited influence on $r_{\gamma}$. All three plots suggest that $R_{0}=1$ but this has not been proved so far even for $H_{0}^{A}$, the Laplacian on the unit disc!

\section{References}

[AhBo] Y. Aharonov, D. Bohm, Significance of Electromagnetic Potentials in the Quantum Theory. Phys. Rev. II 115 (1959), 485-491

[AL] M. Aizenman, E. Lieb, On semiclassical bounds for eigenvalues of Schrödinger operators. Phys. Lett. A 66 (1978), no. 6, 427-429.

[AbSt] M. Abramowitz, I. A. Stegun, Handbook of mathematical functions withs formulas, graphs, and mathematical tables, National Bureau of Standards Applied Mathematics Series 55, Washington D.C., 1964.

[BaEvLe] A. A. Balinsky, W. D. Evans, R. T. Lewis, On the number of negative eigenvalues of Schrödinger operators with an Aharonov-Bohm magnetic field. Proc. R. Soc. Lond. A 457 (2001) 2481-2489

[B1] F. A. Berezin, Covariant and contravariant symbols of operators [Russian]. Math. USSR Izv. 6 (1972), 1117-1151.

[B2] F. A. Berezin, Convex functions of operators [Russian]. Mat. Sb. 88 (1972), 268-276.

[EkF] T. Ekholm, R. L. Frank, On Lieb-Thirring inequalities for Schrödinger operators with virtual level. Comm. Math. Phys. 264 (2006), no. 3, 725-740.

[ELo] L. Erdős, M. Loss, private communication.

[ELoV] L. Erdős, M. Loss, V. Vougalter, Diamagnetic behavior of sums of Dirichlet eigenvalues. Ann. Inst. Fourier 50 (2000), 891-907. 
[FLoW] R. L. Frank, M. Loss, T. Weidl, in preparation.

[H] A. M. Hansson, On the spectrum and eigenfunctions of the Schrödinger operator with Aharonov-Bohm magnetic field. Int. J. Math. Math. Sci. 23 (2005), 3751-3766.

[HuS] D. Hundertmark, B. Simon, A diamagnetic inequality for semigroup differences. J. Reine Angew. Math. 571 (2004), 107-130.

[La] A. Laptev, Dirichlet and Neumann eigenvalue problems on domains in Euclidean spaces. J. Funct. Anal. 151 (1997), no. 2, 531-545.

[LaSa] A. Laptev, Yu. Safarov, A generalization of the Berezin-Lieb inequality. Amer. Math. Soc. Transl. (2) 175 (1996), 69-79.

[LaW1] A. Laptev, T. Weidl, Sharp Lieb-Thirring inequalities in high dimensions. Acta Math. 184 (2000), no. 1, 87-111.

[LaW2] A. Laptev, T. Weidl, Recent results on Lieb-Thirring inequalities. Journées "Équations aux Dérivées Partielles" (La Chapelle sur Erdre, 2000), Exp. No. XX, Univ. Nantes, Nantes, 2000.

[LiY] P. Li, S.-T. Yau, On the Schrödinger equation and the eigenvalue problem. Comm. Math. Phys. 88 (1983), 309-318.

[L] E. H. Lieb, The classical limit of quantum spin systems. Comm. Math. Phys. 31 (1973), 327-340.

[LT] E. H. Lieb, W. Thirring, Inequalities for the moments of the eigenvalues of the Schrödinger Hamiltonian and their relation to Sobolev inequalities. Studies in Mathematical Physics, 269-303. Princeton University Press, Princeton, NJ, 1976.

[Lu] Y. L. Luke, Integrals of Bessel Functions, McGraw-Hill, New York-LondonToronto, 1962.

[MOR] M. Melgaard, E.-M. Ouhabaz, G. Rozenblum, Negative discrete spectrum of perturbed multivortex Aharonov-Bohm Hamiltonians. Ann. Henri Poincaré 5 (2004), no. 5, 979-1012. Erratum: ibid. 6 (2005), no. 2, 397-398.

[P] G. Pólya, On the eigenvalues of vibrating membranes. Proc. London Math. Soc. 11 (1961), 419-433.

[ReS] M. Reed, B. Simon, Methods of Modern Mathematical Physics, vol. 4. Academic Press, New York-London, 1978.

[Ru] S. N. M. Ruijsenaars, The Aharonov-Bohm effect and scattering theory, Ann. Physics 146 (1983), no. 1, 1-34.

[S] B. Simon, Trace ideals and their applications, second ed. Mathematical Surveys and Monographs 120, American Mathematical Society, Providence, RI, 2005.

[Ti] E. C. Titchmarsh, Theory of Fourier Integrals, second ed. Oxford University Press, Oxford, 1948.

Department of Mathematics, School of Engineering Sciences, Royal Institute of Technology, 10044 Stockholm, Sweden

E-mail address: $\{$ rupert, anhan\}@math.kth.se 

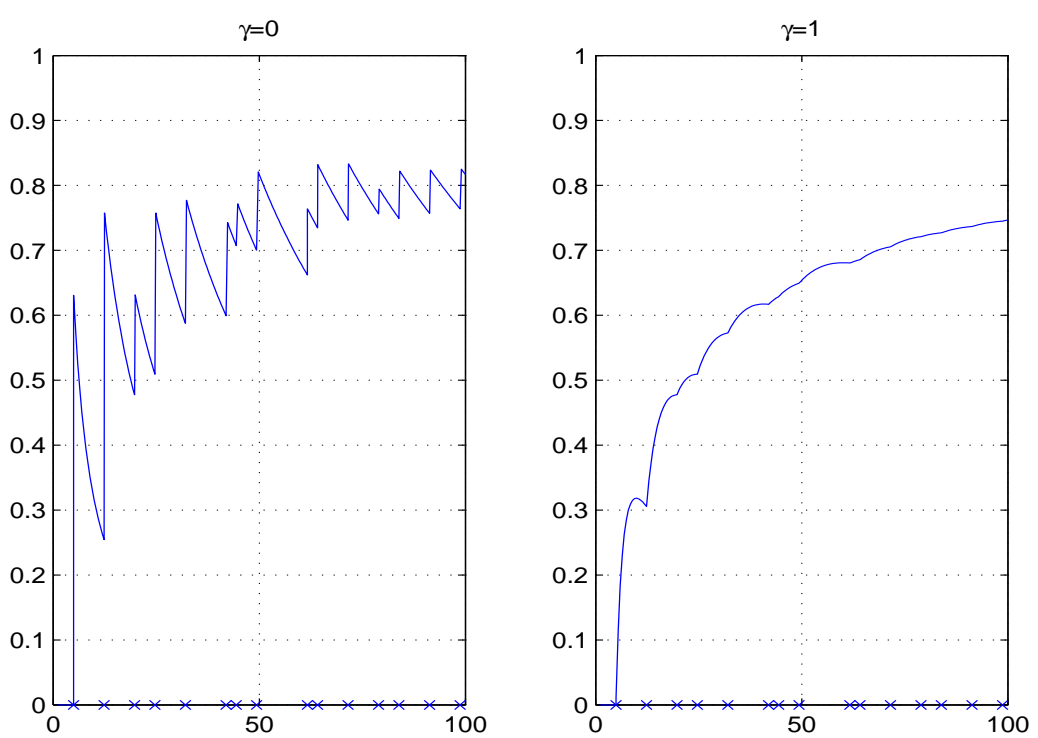

FiguRE 1. $r_{\gamma}$ for $H_{0}^{B}$ near the bottom of the spectrum
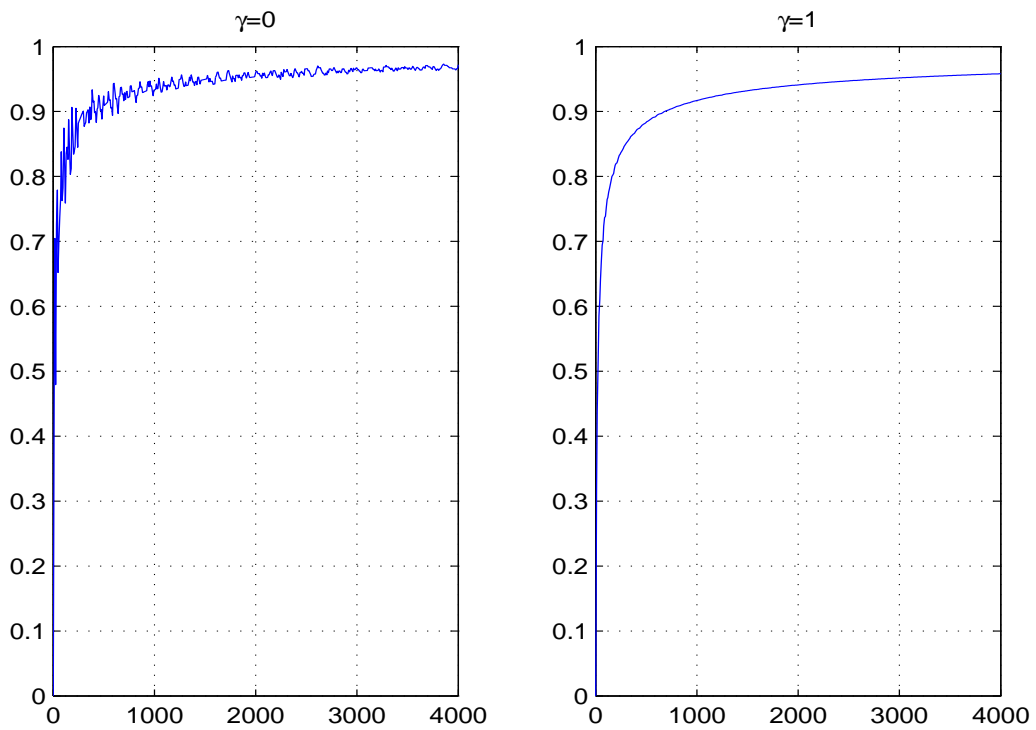

FiguRE 2. $r_{\gamma}(\Lambda)$ for $H_{0}^{B}$ for large $\Lambda$ 


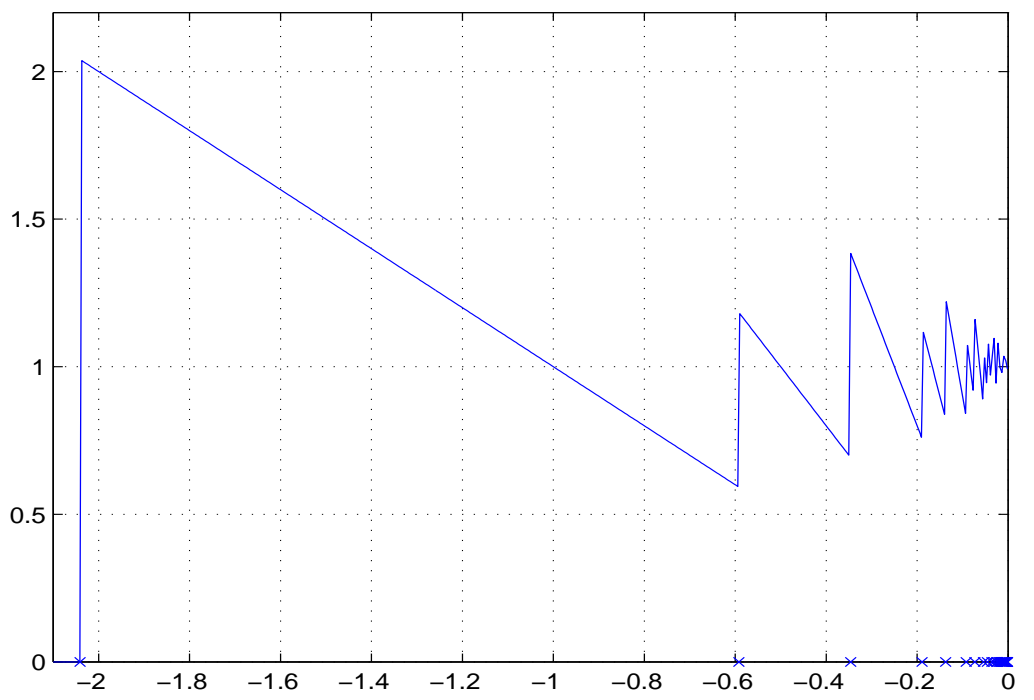

FiguRE 3. $r_{0}$ for an operator with $R_{0}>1$

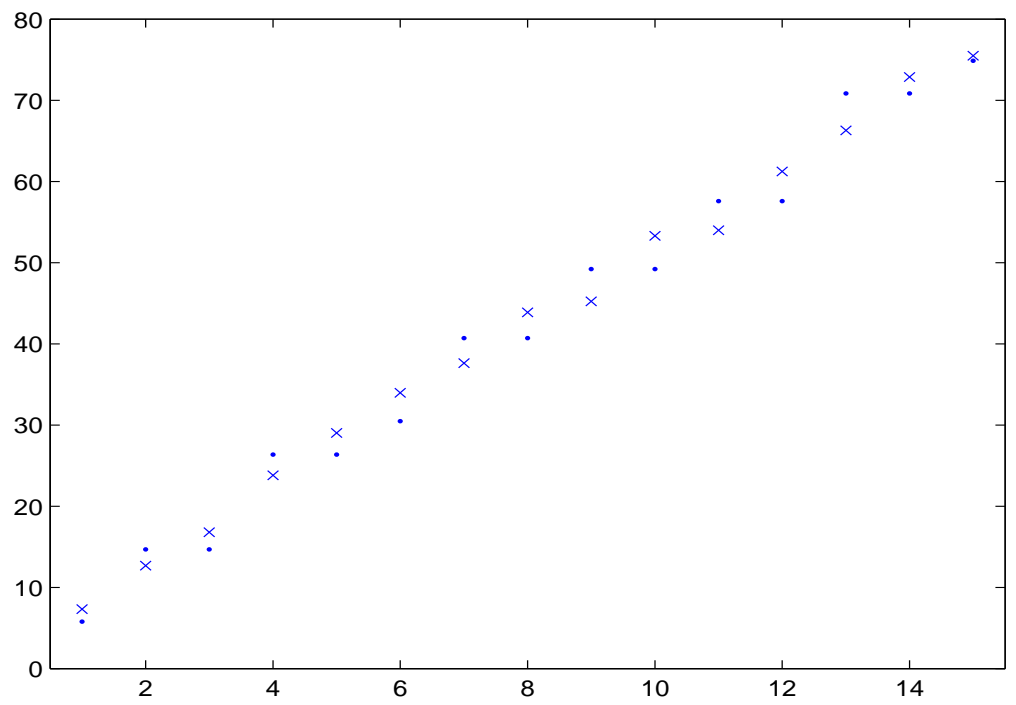

Figure 4. Lowest eigenvalues of $H_{0}^{A}(\cdot)$ and of $H_{0.2}^{A}(\times)$ 


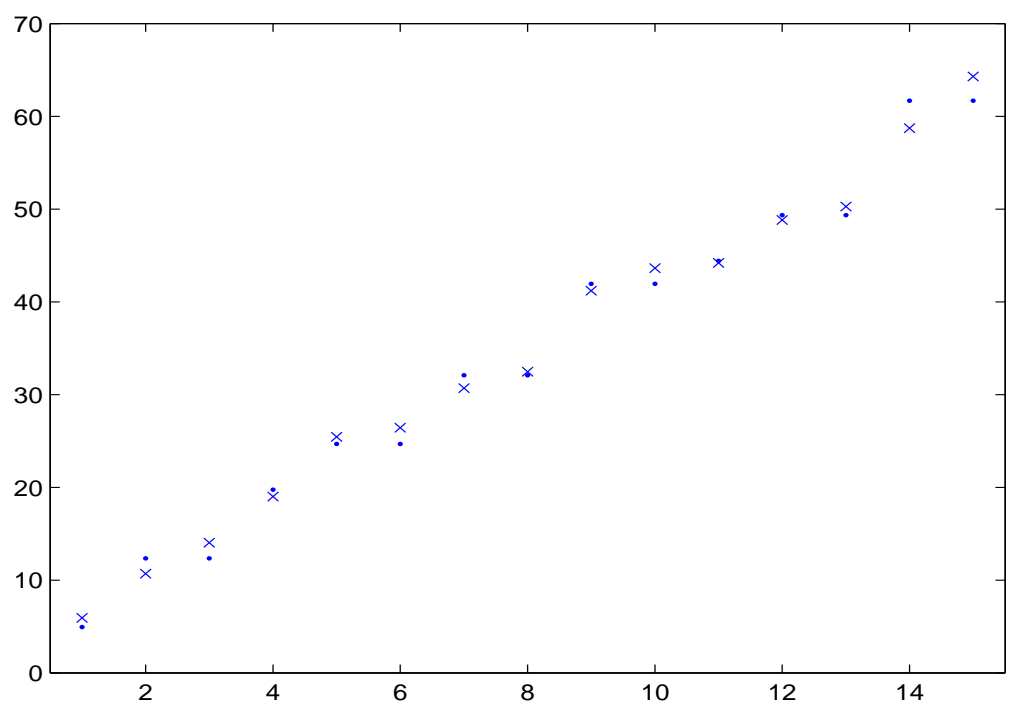

Figure 5. Lowest eigenvalues of $H_{0}^{B}(\cdot)$ and of $H_{0.2}^{B}(\times)$

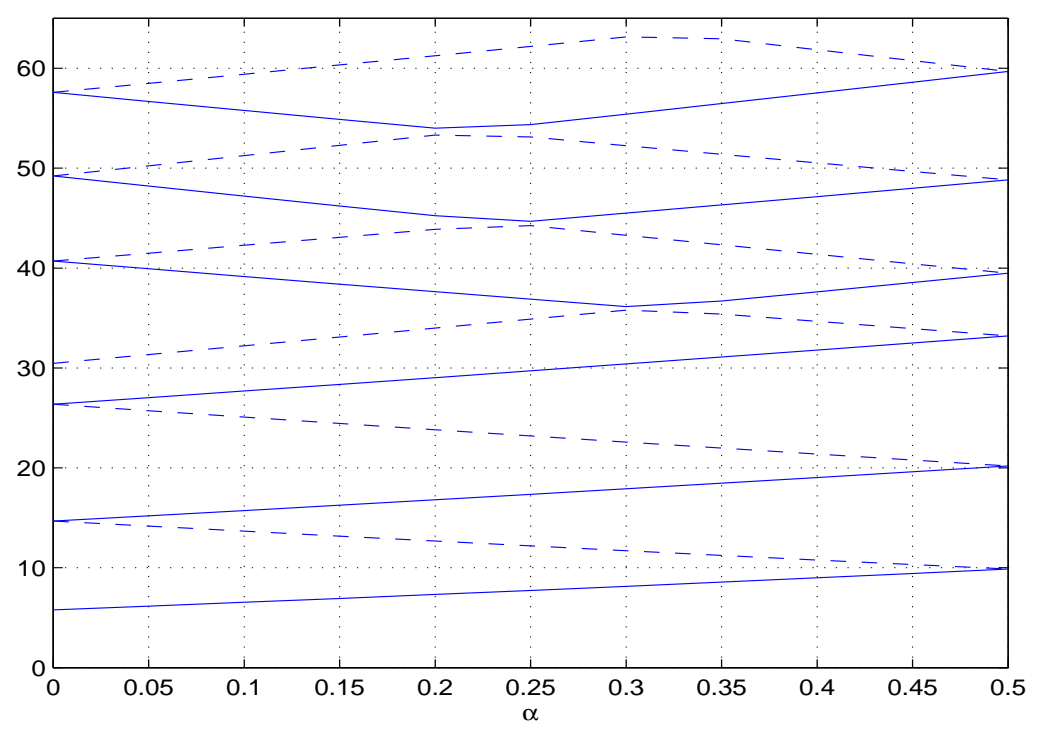

Figure 6. Lowest eigenvalues of $H_{\alpha}^{A}$ as function of $\alpha$ 

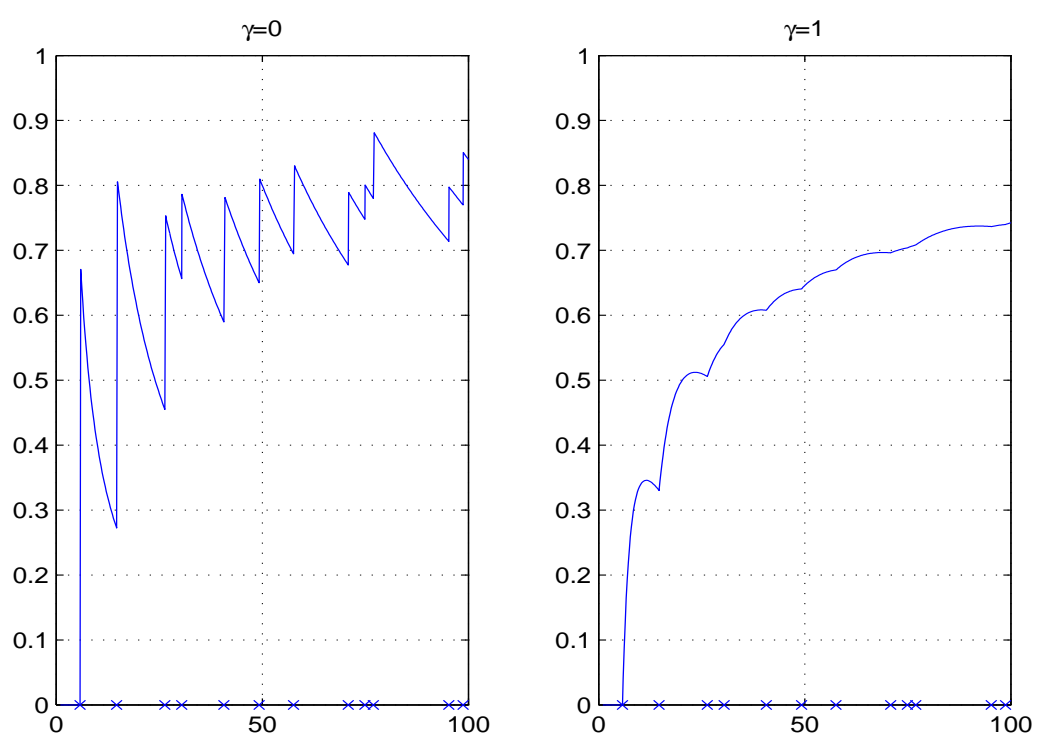

FiguRE $7 . r_{\gamma}$ for $H_{0}^{A}$ near the bottom of the spectrum
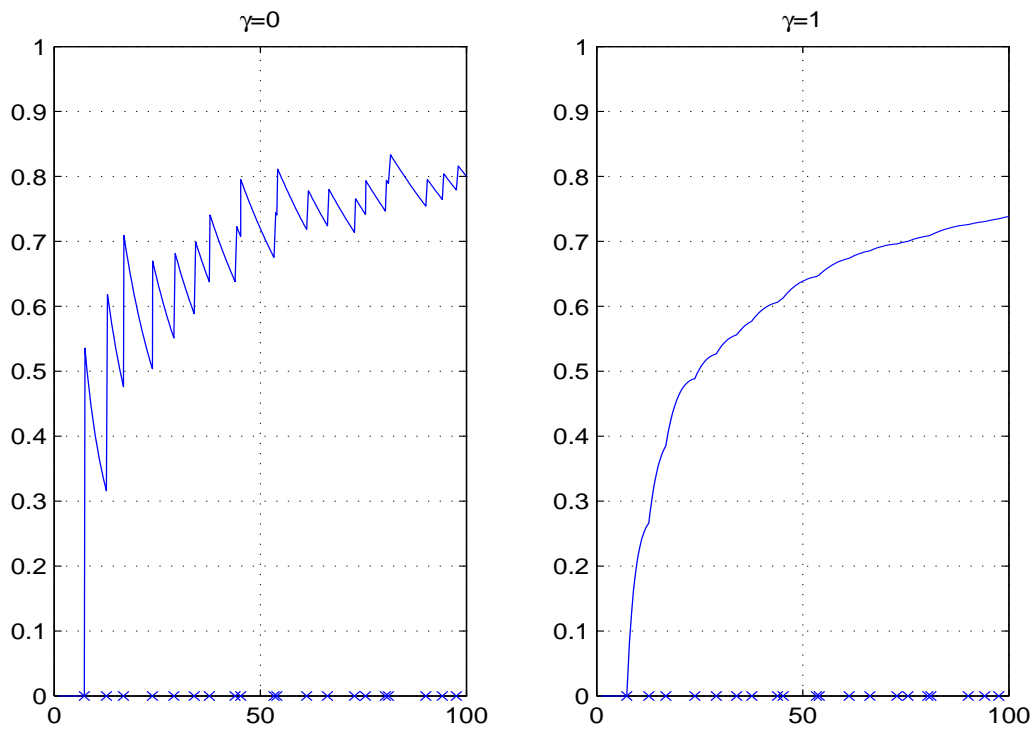

FiguRE 8. $r_{\gamma}$ for $H_{0.2}^{A}$ near the bottom of the spectrum 

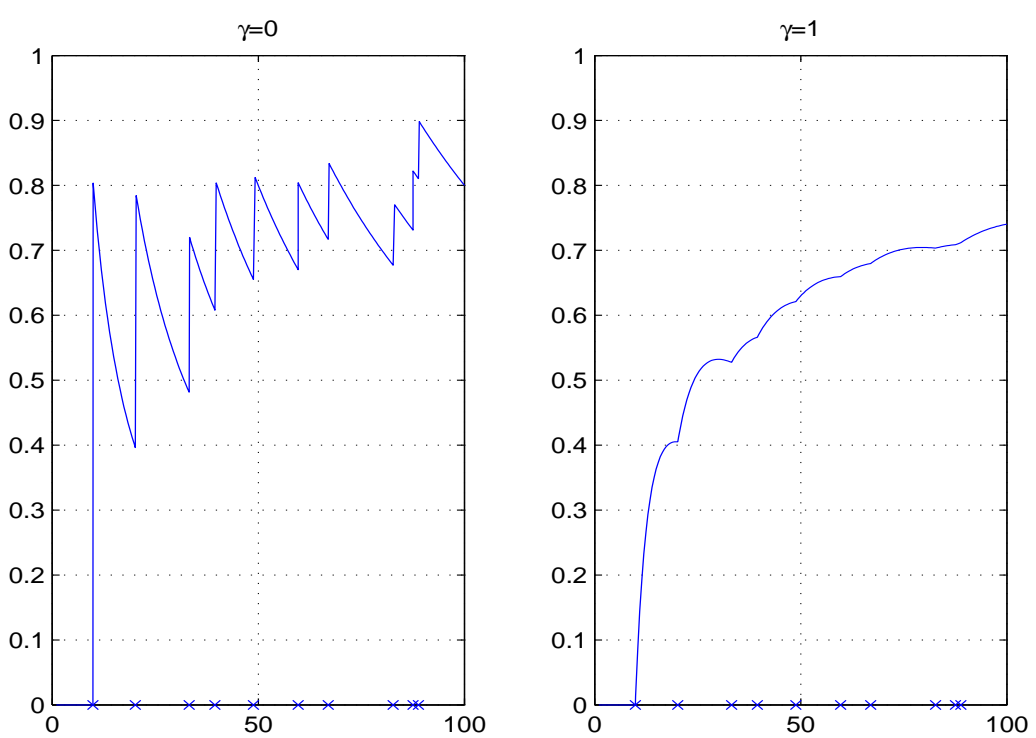

Figure $9 . r_{\gamma}$ for $H_{0.5}^{A}$ near the bottom of the spectrum
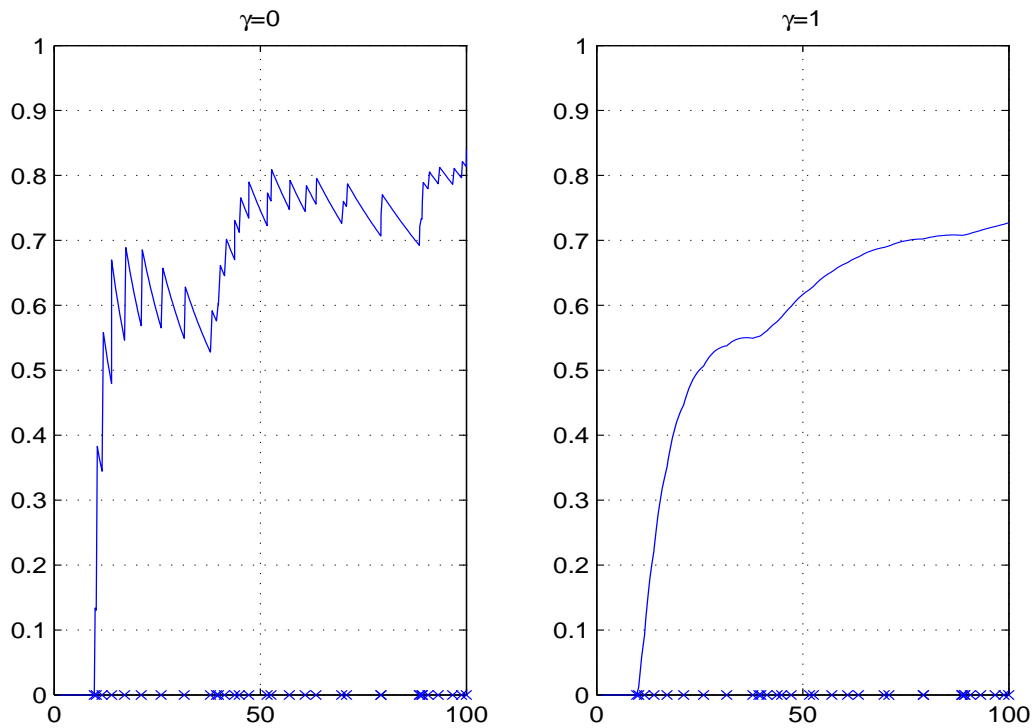

Figure 10. $r_{\gamma}$ for $H_{0}^{D}$ near the bottom of the spectrum 